\title{
Observation of multimodal interference in millimeter-long polymer optical fibers
}

\author{
Yosuke Mizuno $^{1 a)}$, Sonoko Hagiwara ${ }^{1}$, Natsuki Matsutani ${ }^{1}$, Kohei Noda ${ }^{1}$, Heeyoung Lee $^{1}$, and Kentaro Nakamura ${ }^{1}$
}

\begin{abstract}
We observe multimodal interference in single-mode-multimodesingle-mode sensors comprising short polymer optical fibers (POFs) with lengths from $100 \mathrm{~mm}$ down to $7 \mathrm{~mm}$. Characteristic spectral peaks/dips are observed not in the conventionally used telecom band but around $1000 \mathrm{~nm}$. We find that the dip wavelength depends on temperature but that the sensitivity is much lower than those obtained in the longer wavelength range when longer POFs are used. We discuss the possibility that, even with the reduced sensitivity, our success in observing multimodal interference in millimeter-long optical fibers will be a basis toward the combined use of frequency and intensity information in conventional fiber-optic single-point sensors for discriminative measurement of multiple physical parameters.

Keywords: optical fiber sensors, multimodal interference Classification: Optical hardware
\end{abstract}

\section{Introduction}

Sensing technology of strain and temperature using optical fibers has been an active area of research for many decades, and a wide variety of configurations have been developed thus far. They operate based on fiber Bragg gratings $[1,2,3]$, long-period gratings [4, 5], Brillouin scattering $[6,7,8,9]$, Raman scattering $[10,11]$, optical interference $[12,13,14,15,16,17,18,19,20,21,22,23,24]$, and many others. Among numerous types of interference-based sensors, those based on intermodal interference in multimode fibers (MMFs) have recently attracted a considerable attention owing to their system simplicity and cost efficiency. Of all the multimodal-interference-based sensors previously developed, which are well summarized in Table 1 in Ref. [12], the most widely used configuration is what is called a "single-mode-multimode-single-mode" (SMS) structure $[13,14,15,16,17,18,19,20]$, in which two single-mode fibers (SMFs) sandwich an MMF.

To date, the strain and temperature sensitivities of various SMS sensors have been extensively investigated. When a 1.8-m-long silica graded-index (GI-) MMF was used, a strain sensitivity of $+18.6 \mathrm{pm} / \mu \varepsilon$ and a temperature sensitivity of $+58.5 \mathrm{pm} /{ }^{\circ} \mathrm{C}$ were obtained at $1550 \mathrm{~nm}$ [13]. Note that the sensitivities are known to be strongly dependent on optical wavelength; not only their absolute values but also their signs depend on a so-called critical wave-

\footnotetext{
${ }^{1}$ Institute of Innovative Research, Tokyo Institute of Technology, Yokohama 226-8503, Japan

a)ymizuno@sonic.pi.titech.ac.jp
}

DOI: 10.1587/elex.16.20190135

Received March 7, 2019

Accepted March 26, 2019

Publicized April 10, 2019

Copyedited April 25, 2019 length [14], which varies depending on the material and structure of the SMS sensors. As silica glass fibers are relatively fragile and cannot withstand large strain, to extend the strain dynamic range, a 160 -mm-long polymethyl methacrylate (PMMA)-based step-index polymer optical fiber (POF) was used as an MMF, leading to a strain sensitivity of $-1.72 \mathrm{pm} / \mu \varepsilon$ and a temperature sensitivity of $-56.8 \mathrm{pm} /{ }^{\circ} \mathrm{C}$ at $1570 \mathrm{~nm}$ [15]. However, the propagation loss of PMMA-based POFs at telecom wavelength is extremely high $\left(\gg 1 \times 10^{5} \mathrm{~dB} / \mathrm{km}\right)$. To tackle this issue, we have recently implemented SMS-based strain and temperature sensors using perfluorinated (PF) GI-POFs [16, $17,18,19,20,21,22]$, which are the only POFs with a relatively low propagation loss even at telecom wavelength ( $\sim 250 \mathrm{~dB} / \mathrm{km}$ at $1550 \mathrm{~nm} ; \sim 50 \mathrm{~dB} / \mathrm{km}$ at $1300 \mathrm{~nm})$. Using a PFGI-POF with a length of $1.0 \mathrm{~m}$ and a core diameter of $62.5 \mu \mathrm{m}$, we obtained a strain sensitivity of $-111.8 \mathrm{pm} / \mu \varepsilon$ and a temperature sensitivity of $+49.8 \mathrm{~nm} /{ }^{\circ} \mathrm{C}$ at $1300 \mathrm{~nm}$ [16]; these large absolute values suggest that PFGI-POFbased SMS sensors are extremely sensitive to strain and temperature compared with other SMS sensors composed of silica MMFs or PMMA-POFs.

To enhance the usability of the PFGI-POF-based SMS sensors, instead of a conventional two-end-access (or transmissive) configuration, we have implemented a single-endaccess (or reflectometric) configuration and proved its appropriate sensing operation [19]. We have also experimentally clarified that the strain and temperature sensitivities do not largely depend on the length of a POF, when the length is $>300 \mathrm{~mm}$ [19]. If this trend holds true even when the POF length is much shorter than $300 \mathrm{~mm}$, we may be able to develop a fiber-tip-type local-temperature sensor with a high sensitivity (note that, as it is difficult to directly use such a short POF attached at the fiber tip for strain sensing, hereafter we focus on temperature sensing). However, no previous reports have verified whether multimodal interference can be observed in extremely short POFs (not in silica MMFs, either); the influence of the POF length on the temperature sensitivity also remains unclarified.

In this work, we make an attempt to observe multimodal interference in SMS sensors using short PFGI-POFs with lengths from $100 \mathrm{~mm}$ down to $7 \mathrm{~mm}$. We show that spectral peaks/dips induced by multimodal interference are not observed in the conventionally used wavelength range (near the telecom band) but that they can be observed in the shorter wavelength range around $1000 \mathrm{~nm}$. In this range, the dip wavelengths are clearly dependent on temperature, but the sensitivities are much lower than those of longer 
POFs measured in the longer wavelength range. Thus, it appears to be difficult to fabricate fiber-tip-type temperature sensors with high sensitivities by this method, but our finding that multimodal interference can be observed even in millimeter-long optical fibers will add a new functionfrequency-based sensing function-to some of the conventional intensity-based single-point fiber sensors.

\section{Principle}

A conventional transmissive SMS structure is composed of an MMF sandwiched by two SMFs. When the output of a broadband light source is injected into one of the SMFs, the transmitted light exhibits multiple characteristic dips (or peaks) in its spectrum, induced by multimodal interference. Refer to previous literatures [19, 23, 24] for its detailed operating mechanism. The intervals of the neighboring dips are inconstant both in wavelength and frequency. When strain or temperature change is applied to the MMF, these dips shift to longer or shorter wavelengths. The dependence coefficients including their signs are determined by a critical wavelength $\lambda_{\mathrm{C}}$ [14]. Under the assumption that the MMF and the SMFs are axially aligned, the signs become opposite between the wavelength ranges shorter and longer than $\lambda_{\mathrm{C}}$, and the absolute values become larger when the dips are closer to $\lambda_{\mathrm{C}}$. Thus, though an exception based on spectral correlation has been reported [19], we basically need to select one of the dips suitable for our specific applications. If a higher sensitivity is preferable, the dip nearest to $\lambda_{\mathrm{C}}$ should be used; while if a wider strain/temperature dynamic range is preferable, it may not be the best dip to be selected. To date, when the MMF is a PFGI-POF, a dip around $1350 \mathrm{~nm}$ has been often used for its higher sensitivity [16, 19, 20].

An SMS structure can be implemented using an MMF and a single SMF in a reflectometric manner, where the Fresnel reflection at the open end of the MMF is exploited. Using the same PFGI-POF, we compared the sensing performances between the transmissive and reflectometric configurations at $\sim 1350 \mathrm{~nm}$, and found that the strain and temperature sensitivities are almost identical regardless of the configurations [19]. This fact indicates that the sensitivities do not change when the POF length is doubled. We then investigated the sensitivity dependence on the POF length $(>300 \mathrm{~mm})$ using the transmissive configuration, and clarified that the sensitivities do not exhibit a clear dependence on the POF length [19]. However, multimodal interference has not been observed in POFs or silica MMFs much shorter than $300 \mathrm{~mm}$; the shortest MMF in which multimodal interference has been observed is the aforementioned PMMA-POF with a length of $160 \mathrm{~mm}$ (actually, limited by its high propagation loss) [15]. Considering that the visibility of the spectral dips/peaks tends to be reduced with decreasing fiber length (refer to the discussion on a socalled refocus-length) [21], it is a challenge to observe multimodal interference in a millimeter-long POF.

\section{Experimental setup}

The MMFs used in the experiment were PFGI-POFs [25] with a core diameter of $50 \mu \mathrm{m}$, a cladding diameter of $70 \mu \mathrm{m}$, and a overcladding diameter of $490 \mu \mathrm{m}$. The core and cladding layers were doped and undoped polyperfluorobutenylvinyl ether, respectively. The refractive indices at the center of the core and in the cladding layer were approximately 1.356 and 1.342 , respectively, which do not largely depend on optical wavelength. An overcladding layer was composed of polycarbonate, which was coated on the cladding to improve the load-bearing capability. The lengths of the POFs were $100 \mathrm{~mm}, 40 \mathrm{~mm}$, and $7 \mathrm{~mm}$.

The experimental setup of the SMS-based temperature sensor using the PFGI-POF is depicted in Fig. 1. The output of a supercontinuum source (SCS) covering a wide band from 460 to $2000 \mathrm{~nm}$ (output power: $75 \mathrm{~mW}$ ) was injected into the POF via a 2.5-m-long SMF. The light transmitted through the POF was injected into another 3.0m-long SMF, and its spectrum was observed using an optical spectrum analyzer (OSA; bandwidth: 600-1700 $\mathrm{nm})$. The optical couplings between the POF and the SMFs were performed using a butt-coupling technique [26]. As the POF was short, its whole length was, along with the couplings to SMFs, placed in a thermostatic chamber and heated from room temperature $\left(\sim 20^{\circ} \mathrm{C}\right)$ up to $\sim 55^{\circ} \mathrm{C}$. The whole setup was fixed during all the measurements, leading to a negligible change in the polarization state.

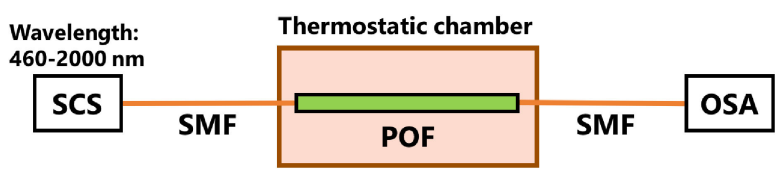

Fig. 1. Experimental setup for investigating the temperature sensitivity of single-mode-multimode-single-mode sensors involving short polymer optical fibers (POFs) with different lengths. OSA, optical spectrum analyzer; SCS, supercontinuum source; SMF, single-mode fiber.

\section{Experimental results}

The measured spectra of the SCS output and the light transmitted through the 100-mm-long POF around 1350 $\mathrm{nm}$ are shown in Fig. 2(a). The result with a 1.0-m-long POF is also included for comparison. When the POF length was $1.0 \mathrm{~m}$, clear dips caused by multimodal interference were observed at the wavelengths where no dips were observed in the SCS output. However, when the POF length was $100 \mathrm{~mm}$, no clear dips were observed in this range; we confirmed that, when the POF was heated, almost no spectral change was observed. This is natural considering the aforementioned fact that the visibility of the peaks/dips tends to be reduced with decreasing length of the POF. Then, we scanned the wavelength range to be monitored from 600 to $1700 \mathrm{~nm}$, and found that a characteristic spectral pattern involving multiple dips can be observed around $1000 \mathrm{~nm}$ even when the POF length was $100 \mathrm{~mm}$. The locations of the dips were not identical to those in the SCS output. In addition, the intervals between the neighboring dips were not constant in the frequency domain, which indicates that this pattern does not originate from a fiber Fabry-Perot cavity [27, 28]. Thus, we conclude that this pattern was caused by multimodal interference. 


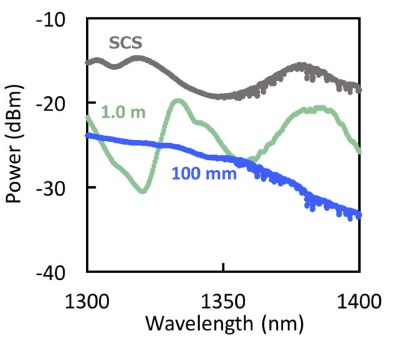

(a)

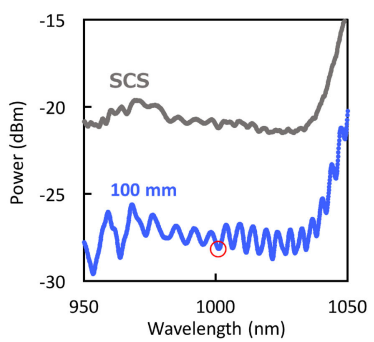

(b)
Fig. 2. Measured optical spectra of the supercontinuum source (SCS) output and the light transmitted through the POFs. (a) When the POF lengths were $1.0 \mathrm{~m}$ and $100 \mathrm{~mm}$; around $1350 \mathrm{~nm}$. (b) When the POF length was $100 \mathrm{~mm}$; around $1000 \mathrm{~nm}$. The red circle indicates the dip used for investigating the temperature dependence.

For all the POFs shorter than $100 \mathrm{~mm}$, similar spectral patterns were observed around $1000 \mathrm{~nm}$.

Subsequently, we measured the temperature dependence of the one of the dips around $1000 \mathrm{~nm}$ (the one closest to $1000 \mathrm{~nm}$; indicated by a red circle in Fig. 2(b)) when the POF length was $100 \mathrm{~mm}$; see Appendix for the validity of selecting this dip. The spectral dependence on temperature is shown in Fig. 3(a). All the measured spectra were vertically shifted and normalized so that the dip power became 0 . With increasing temperature, the dip shifted to longer wavelength. Then, we plotted the dip wavelength as a function of temperature (Fig. 3(b)). The dependence was almost linear in this range, and the dependence coefficient was $83.5 \mathrm{pm} /{ }^{\circ} \mathrm{C}$. We also measured the temperature dependencies of the spectral dips and the dip wavelengths when POFs with lengths of $40 \mathrm{~mm}$ and $7 \mathrm{~mm}$ were used (Figs. 3(c)-(f)). Regardless of the POF lengths, similar trends were obtained, and the dependence coefficients were $80.3 \mathrm{pm} /{ }^{\circ} \mathrm{C}$ and $93.8 \mathrm{pm} /{ }^{\circ} \mathrm{C}$ for the $40-\mathrm{mm}$-long and $7-$ mm-long POFs, respectively. These values are approximately 100 times smaller than those measured around $1350 \mathrm{~nm}$ using POFs longer than $300 \mathrm{~mm}$ [19], probably because of the different wavelength ranges where spectral dips can be observed. Another finding is that, when a POF is shorter than $100 \mathrm{~mm}$, the temperature sensitivity did not largely depend on the POF length, which is the same trend as that of longer POFs. We can interpret that the sensitivity does not largely depend on the POF length as long as the wavelength range used for the measurement is identical.

\section{Discussion}

When the POF length was shorter than $100 \mathrm{~mm}$, the obtained temperature sensitivity was much lower than those with longer POFs. As the most important advantage of SMS-based temperature sensors using PFGI-POFs is their high sensitivity, it is not beneficial to fabricate a fiber-tiptype temperature sensor using such a short POF. However, our success in observing multimodal interference in a millimeter-long MMF, even with a low temperature sensitivity, has enormous significance in terms of adding a new function to intensity-based fiber-optic point sensors. One example is what they call hetero-core sensors [29, 30], which are composed of a short small-core SMF sandwiched between two larger-core SMFs. They are used to

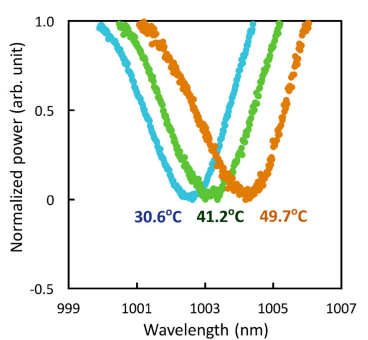

(a)

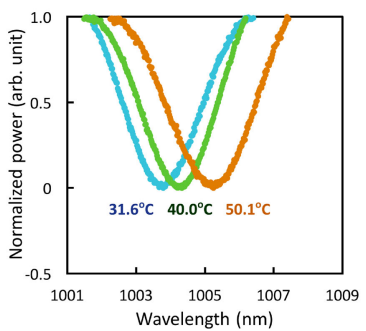

(c)

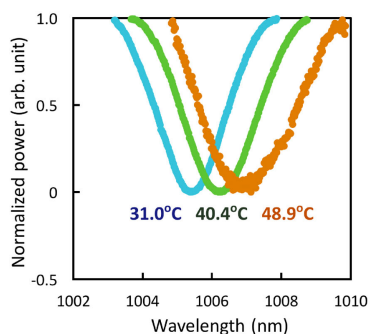

(e)

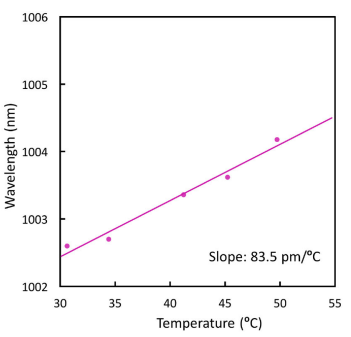

(b)

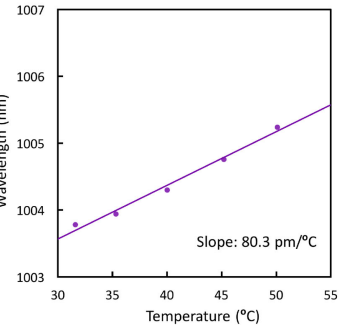

(d)

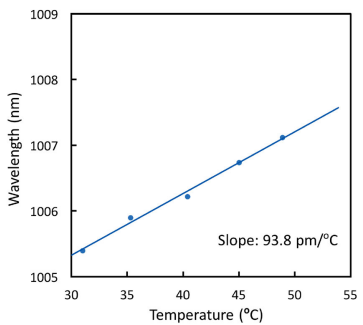

(f)
Fig. 3. Measured temperature dependencies of the normalized spectra and the dip wavelengths. (a) spectrum, 100-mm-long POF; (b) wavelength, 100-mm-long POF; (c) spectrum, 40-mm-long POF; (d) wavelength, 40-mm-long POF; (e) spectrum, 7-mm-long POF; (f) wavelength, 7-mm-long POF. The solid lines in (b), (d), and (f) are linear fits.

measure various physical parameters such as deformation, pressure [29], and acidity [30], based on optical loss (i.e., intensity information) induced at the hetero-core parts. As multiple propagation modes including cladding modes exist in a small-core SMF, multimodal interference could also be observed in this configuration. If frequency-based sensing function is added to the conventional intensity-based operating mechanism of hetero-core sensors, discriminative sensing of multiple parameters will be feasible. Another example is swelling-clad-based POF sensors [31, 32], in which claddings are partially removed and replaced with swelling polymer. Based on optical loss, some parameters such as ambient humidity [31] and alkane [32] can be measured. As the number of the propagation modes at the cladding-removed part is different from those of other parts, multimodal interference could also be observed in this kind of sensor. Thus, our finding will be a basis for the combined use of intensity and frequency information in conventional single-point sensors.

\section{Conclusion}

Multimodal interference was observed, for the first time to the beset of our knowledge, in SMS sensors using short PFGI-POFs with lengths of $100 \mathrm{~mm}, 40 \mathrm{~mm}$, and $7 \mathrm{~mm}$. Spectral dips were observed not in the conventionally used 
telecom band but in the shorter wavelength range around $1000 \mathrm{~nm}$. The dip wavelengths showed a clear dependence on temperature, but regardless of the POF length, the sensitivities were approximately 100 times lower than the values previously reported with longer POFs in the longer wavelength range. This result indicates that it is not beneficial to fabricate fiber-tip-type temperature sensors based on multimodal interference in short POFs because of the difficulty in obtaining high sensitivities. However, we anticipate that the observation of multimodal interference in millimeter-long optical fibers will open up a new way to adding a frequency-based sensing function to some of the conventional intensity-based single-point sensors.

\section{Acknowledgments}

This work was supported by JSPS KAKENHI Grant Numbers $17 \mathrm{H} 04930$ and $17 \mathrm{~J} 07226$ and by a research grant from the Fujikura Foundation.

\section{Appendix}

When a $100-\mathrm{mm}$-long POF was used, the spectrum of the transmitted light had more than 10 dips around $1000 \mathrm{~nm}$, as shown in Fig. 2(b). We selected a particular dip and investigated its temperature dependence. However, as the temperature sensitivity has been reported to depend on optical wavelength [14], it may not be fair to evaluate the sensing performance using only a single dip. Here, we measure the difference in the temperature sensitivities of the different dips. Fig. A·1(a) shows the temperature dependence of the spectrum of the transmitted light around $1000 \mathrm{~nm}$ when the 100 -mm-long POF was used. We selected two dips (located at $\sim 962 \mathrm{~nm}$ and $\sim 1045 \mathrm{~nm}$ when temperature was $52.1{ }^{\circ} \mathrm{C}$; indicated by red circles) and measured their wavelength dependencies on temperature,

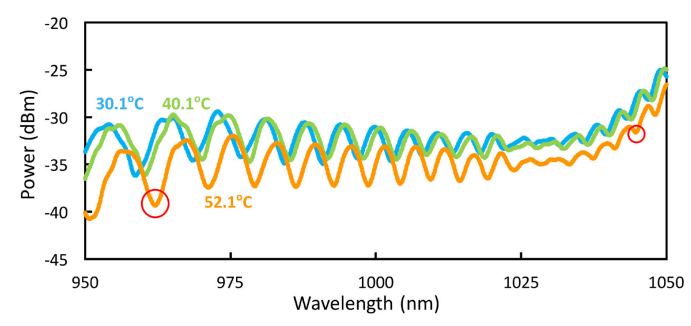

(a)

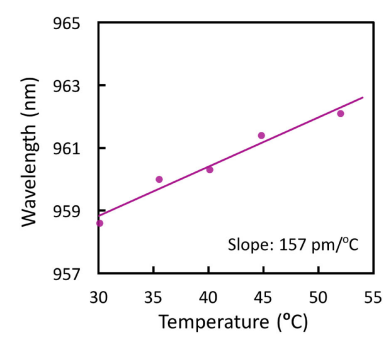

(b)

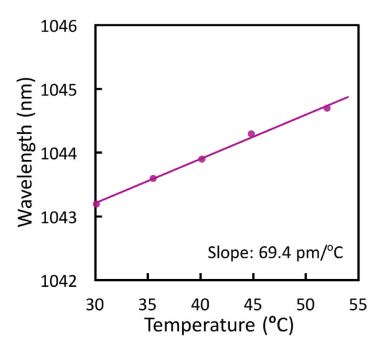

(c)
Fig. A.1. (a) Temperature dependence of the spectrum of the transmitted light around $1000 \mathrm{~nm}$ when the POF length was $100 \mathrm{~mm}$. The red circles indicate the measured dips. (b, c) The temperature dependencies of the two dip wavelengths. The solid lines are linear fits. as shown in Figs. A·1(b) and (c). The dependencies were both almost linear, and the coefficients of the dips at shorter and longer wavelengths were $157 \mathrm{pm} /{ }^{\circ} \mathrm{C}$ and $69.4 \mathrm{pm} /{ }^{\circ} \mathrm{C}$, respectively. Although the former is approximately 2.3 times higher than the latter, this result suggests that the selection of an arbitrary dip does not alter the conclusion that the obtained sensitivity around $1000 \mathrm{~nm}$ is by far lower than those in the telecom band.

\section{References}

[1] A. D. Kersey, et al.: "Fiber grating sensors," J. Lightw. Technol. 15 (1997) 1442 (DOI: 10.1109/50.618377).

[2] B.-O. Guan, et al.: "Simultaneous strain and temperature measurement using a superstructure fiber Bragg grating," IEEE Photon. Technol. Lett. 12 (2000) 675 (DOI: 10.1109/68.849081).

[3] Y. Zhang and W. Yang: "Simultaneous precision measurement of high temperature and large strain based on twisted FBG considering nonlinearity and uncertainty," Sens. Actuators A 239 (2016) 185 (DOI: 10.1016/j.sna.2016.01.012).

[4] V. Bhatia and A. M. Vangsarkar: "Optical fiber long-period grating sensors," Opt. Lett. 21 (1996) 692 (DOI: 10.1364/OL.21.000692).

[5] Y. P. Wang, et al:: "Highly sensitive long-period fiber-grating strain sensor with low temperature sensitivity," Opt. Lett. 31 (2006) 3414 (DOI: 10.1364/OL.31.003414).

[6] Y. Dong, et al.: "High-spatial-resolution fast BOTDA for dynamic strain measurement based on differential double-pulse and secondorder sideband of modulation," IEEE Photon. J. 5 (2013) 2600407 (DOI: 10.1109/JPHOT.2013.2267532).

[7] K. Hotate and T. Hasegawa: "Brillouin optical-fiber time domain reflectometry," IEICE Trans. Electron. E83-C (2000) 405.

[8] Y. Mizuno, et al.: "Proposal of Brillouin optical correlationdomain reflectometry (BOCDR)," Opt. Express 16 (2008) 12148 (DOI: 10.1364/OE.16.012148).

[9] Y. Mizuno, et al:: "Ultrahigh-speed distributed Brillouin reflectometry," Light Sci. Appl. 5 (2016) e16184 (DOI: 10.1038/ lsa.2016.184).

[10] M. A. Farahani and T. Gogolla: "Spontaneous Raman scattering in optical fibers with modulated probe light for distributed temperature Raman remote sensing," J. Lightw. Technol. 17 (1999) 1379 (DOI: 10.1109/50.779159)

[11] M. N. Alahbabi, et al.: "Simultaneous temperature and strain measurement with combined spontaneous Raman and Brillouin scattering," Opt. Lett. 30 (2005) 1276 (DOI: 10.1364/OL.30. 001276).

[12] O. Frazão, et al:: "Optical fiber refractometry based on multimode interference," Appl. Opt. 50 (2011) E184 (DOI: 10.1364/AO.50. 00E184).

[13] Y. Liu and L. Wei: "Low-cost high-sensitivity strain and temperature sensing using graded-index multimode fibers," Appl. Opt. 46 (2007) 2516 (DOI: 10.1364/AO.46.002516).

[14] S. M. Tripathi, et al.: "Strain and temperature sensing characteristics of single-mode-multimode-single-mode structures," J. Lightw. Technol. 27 (2009) 2348 (DOI: 10.1109/JLT.2008. 2008820).

[15] J. Huang, et al: "Polymer optical fiber for large strain measurement based on multimode interference," Opt. Lett. 37 (2012) 4308 (DOI: 10.1364/OL.37.004308).

[16] G. Numata, et al.: "Ultra-sensitive strain and temperature sensing based on modal interference in perfluorinated polymer optical fibers," IEEE Photon. J. 6 (2014) 6802306 (DOI: 10.1109/JPHOT. 2014.2352637).

[17] G. Numata, et al.: "Strain and temperature sensing based on multimode interference in partially chlorinated polymer optical fibers," IEICE Electron. Express 12 (2015) 20141173 (DOI: 10. 1587/elex.12.20141173).

[18] G. Numata, et al.: "Drastic sensitivity enhancement of temperature sensing based on multimodal interference in polymer optical fibers,” Appl. Phys. Express 8 (2015) 072502 (DOI: 10.7567/ APEX.8.072502). 
[19] T. Kawa, et al.: "Single-end-access strain and temperature sensing based on multimodal interference in polymer optical fibers," IEICE Electron. Express 14 (2017) 20161239 (DOI: 10.1587/elex.14. 20161239).

[20] T. Kawa, et al:: "Temperature sensing based on multimodal interference in polymer optical fibers: Room-temperature sensitivity enhancement by annealing," Jpn. J. Appl. Phys. 56 (2017) 078002 (DOI: 10.7567/JJAP.56.078002).

[21] Y. Mizuno, et al.: "Displacement sensing based on modal interference in polymer optical fibers with partially applied strain," Jpn. J. Appl. Phys. 57 (2018) 058002 (DOI: 10.7567/JJAP.57. 058002).

[22] Y. Mizuno, et al.: "Multimodal interference in perfluorinated polymer optical fibers: Application to ultrasensitive strain and temperature sensing," IEICE Trans. Electron. E101-C (2018) 602 (DOI: 10.1587/transele.E101.C.602).

[23] A. Kumar, et al:: "Transmission characteristics of SMS fiber optic sensor structures," Opt. Commun. 219 (2003) 215 (DOI: 10.1016/ S0030-4018(03)01289-6).

[24] M. Kumar, et al.: "A comparison of temperature sensing characteristics of SMS structures using step and graded index multimode fibers," Opt. Commun. 312 (2014) 222 (DOI: 10.1016/ j.optcom.2013.09.034).

[25] Y. Koike and M. Asai: "The future of plastic optical fiber," NPG Asia Mater. 1 (2009) 22 (DOI: 10.1038/asiamat.2009.2).

[26] Y. Mizuno and K. Nakamura: "Experimental study of Brillouin scattering in perfluorinated polymer optical fiber at telecommunication wavelength," Appl. Phys. Lett. 97 (2010) 021103 (DOI: 10.1063/1.3463038)

[27] Y. Mizuno, et al.: "Fiber-optic interferometry using narrowband light source and electrical spectrum analyzer: Influence on Brillouin measurement," J. Lightw. Technol. 32 (2014) 4734 (DOI: 10.1109/ JLT.2014.2365187).

[28] J. Gallego, et al.: "High-finesse fiber Fabry-Perot cavities: Stabilization and mode matching analysis," Appl. Phys. B 122 (2016) 47 (DOI: 10.1007/s00340-015-6281-z).

[29] M. Nishiyama, et al:: "Unconstrained pulse pressure monitoring for health management using hetero-core fiber optic sensor," Biomed. Opt. Express 7 (2016) 3675 (DOI: 10.1364/BOE.7.003675).

[30] M. Iga, et al.: "Acidity measurements based on a hetero-core structured fiber optic sensor," Sens. Actuators B 96 (2003) 234 (DOI: 10.1016/S0925-4005(03)00530-6).

[31] S. Muto, et al.: "A plastic optical fibre sensor for real-time humidity monitoring," Meas. Sci. Technol. 14 (2003) 746 (DOI: 10.1088/0957-0233/14/6/306).

[32] A. Nakamura, et al.: "Swelling clad-type plastic optical fiber alkane sensor with multi-layer cladding using electrospray deposition method," Proc. SPIE 10323 (2017) 1032329 (DOI: 10.1117/12. 2263258). 